\title{
Transverse horizontal spatial coherence in shallow water
}

\author{
Bo Zhang*, Fenghua Li, Zhenglin Li, Yanjun Zhang, and Jingyan Wang \\ State Key Laboratory of Acoustics, Institute of Acoustics, Chinese Academy of Sciences, 100190 Beijing, China
}

\begin{abstract}
Since the advent of large-aperture array processing, more and more attention has been paid to the sound field correlation, which has fundamental limit to the array gain of spatial coherent signal processing. The two dominant mechanisms that degrade the spatial coherence are normal modes (or multipaths) interference and the environmental variability caused by several relevant oceanographic processes. In the present study, the transverse horizontal spatial coherence of explosive signals has been studied experimentally by a bottom-mounted array in the Northern South China Sea. And the effects of normal mode interference on the transverse horizontal spatial coherence have been analyzed numerically. Expressed in terms of wavelengths, the coherence length is shown to be larger than $170 \lambda / 185 \lambda$ at acoustic frequency $508-640 \mathrm{~Hz} / 80-101 \mathrm{~Hz}$ in shallow water. It is much greater than Carey's shallow-water result $30 \lambda$ estimated from array signal gain after assuming a specific functional form for the coherence (The Journal of the Acoustical Society of America 104, 831 (1998)). It, however, is consistent with Rouseff's modelling result of a coherence length larger than 100ג (The Journal of the Acoustical Society of America 138, 2256 (2015)). Both Carey and Rouseff argue that the transverse horizontal spatial coherence length depends only weakly on range, in direct. In the present study, however, the coherence length is shown to depend highly on source-receiver range, and it fluctuates synchronously with the sound-field intensity while range varies.
\end{abstract}

\section{Introduction}

The spatial correlation of acoustic field is an important characteristic in underwater acoustics. The resolution of a horizontal array can be improved by increasing its aperture. However, the spatial coherence of acoustic field has physical limitations on the performance of array beamforming spatial gain. Generally, an array's coherence length gives the maximum separation between two points for which coherent processing produces useful gain when a distant source is at broadside[1].

Many works have been done on the horizontallongitudinal correlations in the past years[2-7]. However, there are relatively rare results of the transverse horizontal correlation because it is difficult to deploy a large-scale horizontal array. Carey[8] investigated shallow-water experimental data taken from around the world, and estimated transvers horizontal coherence length $L_{c o h}$ from array signal gain after assuming a specific functional form for the coherence. Expressed in terms of wavelengths, the average coherence length $L_{c o h}$

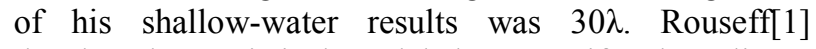
developed a statistical model that quantifies how linear internal waves affects the transverse horizontal $L_{c o h}$, neglecting all other forms of environmental variability. Rouseff concluded that $L_{c o h}$ is strongly dependent on where the source and array are positioned in the water column. As for a range of $20 \mathrm{~km}$, internal wave energy of $400 \mathrm{~J} / \mathrm{m}^{2}$ and a water column of $80 \mathrm{~m}$, the modeling result of $L_{c o h}$ is between $70 \lambda$ and $180 \lambda$ at acoustic frequency higher than $200 \mathrm{~Hz}$ but below $800 \mathrm{~Hz}$, while $L_{c o h}$ is between $220 \lambda$ and $330 \lambda$ below $100 \mathrm{~Hz}$. Both Carey and Rouseff argue that the transverse horizontal spatial coherence length depends only weakly on sourcereceiver range. Zhang[9] investigated the experimental data in the Yellow Sea, and he found that $L_{c o h}$ at the same range fluctuated synchronously with the soundfield intensity while frequency varies.

In this paper, we present the experimental result about the transvers horizontal spatial correlations of the sound field in shallow water by using the experimental data obtained in the Northen South China Sea. And we investigate the transvers coherence in shallow water by combining the normal modes theory with geometry analysis.

\section{Backgrounds}

Fig.1 sketches the scenario of interest. An acoustic source at position $\left(0,0, z_{s}\right)$ is oriented broadside to a distant horizontal line array (HLA) at range $r$ and depth $z_{r}$. The blobs in the figure represent environmental factors that can affect array coherence. These include linear and non-linear internal waves, sea-surface waves, neutrally buoyant intrusions, bottom roughness, and properties of the seabed[1].

Spatial coherence, which describes the similarity between the acoustic fields at two spatially separated points, has no widely accepted definition, but for present

*Corresponding author: zhangbo@mail.ioa.ac.cn 
purposes, the horizontal correlation coefficient of the acoustic fields measured along the HLA is defined as

$$
\begin{aligned}
& \rho(r, \Delta r, \Delta l) \\
& =\max _{\tau} \frac{\int_{-\infty}^{\infty} p(r, l ; t) p(r+\Delta r, l+\Delta l ; t+\tau) d t}{\sqrt{\int_{-\infty}^{\infty} p^{2}(r, l ; t) d t \int_{-\infty}^{\infty} p^{2}(r+\Delta r, l+\Delta l ; t) d t}}
\end{aligned}
$$

where $p(r, l ; t)$ is the sound pressure at $(r, l)$ in time $t$ domain, $\tau$ is the time delay, $\Delta r$ and $\Delta l$ is the longitudinal and transverse separation respectively. Eq. (1) will be used to calculate the correlation coefficient in the following experiment data analysis.

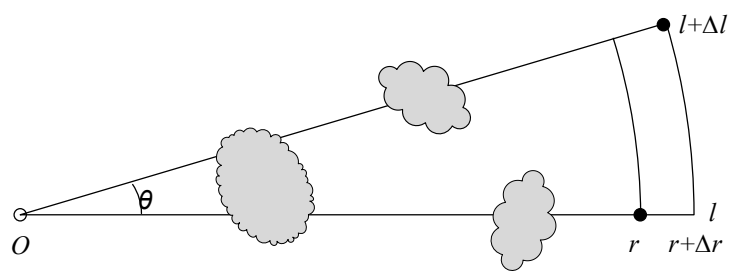

Fig. 1. Array coherence geometry. Source in inhomogeneous ocean oriented at broadside to a distant horizontal array at range $r$. Objective is to analyse correlation between sound pressures at points $(r, l)$ and $(r+\Delta r, l+\Delta l)$.

The acoustic fields with high correlation $\rho=1$ are said to be coherent and $\rho=0$ are referred to as completely incoherent.

On condition that $\Delta r$ is small enough, we have

$$
\begin{gathered}
p(r+\Delta r, l+\Delta l ; t+\tau) \approx p(r, l+\Delta l ; t+\tau) \\
\rho(r, \Delta r, \Delta l) \approx \rho(r, 0, \Delta l)
\end{gathered}
$$

where $\rho(r, 0, \Delta l)$ is defined the transverse horizontal correlation coefficient.

The horizontal separation $\Delta l=L_{c o h}$ such that

$$
\rho\left(r, 0, l+L_{c o h}\right)=\rho(r, 0, l) e^{-1}
$$

defines the transverse horizontal coherence length $L_{c o h}$ as used in this paper.

\section{Experiment descriptions}

\subsection{Experiment setup}

In June of 2017, an experiment was conducted in the Northern South China Sea. As shown in Fig.2, the receiving horizontal line array (HLA) of self-contained hydrophones with synchronous function was bottommounted with a water column of about $100 \mathrm{~m}$. The explosive sources (ES) were oriented broadside to the HLA, which were casted from the experiment ship sailing towards the HLA at a speed of 9 knots. The ship track was almost perpendicular to the isobaths. The predetermined depth of source explosion was $50 \mathrm{~m}$. The ES source level is given in Table 1.

A vertical TD array was deployed near the HLA to measure the temperature profile variability with time. The temperature profiles along the propagation track were measured using XBT.
During the experiment, 46 ESs and 16 XBTs were casted along the propagation track. The ranges of ESs and XBTs to the HLA, as well as the water column depths, are shown in Fig. 3

Table 1. Explosive source level (SL).

\begin{tabular}{|c|c|}
\hline $\begin{array}{c}\text { Frequency } \\
(\mathbf{H z})\end{array}$ & $\begin{array}{c}\text { SL } \\
(\mathbf{d B} \text { re } \mathbf{\mu} \text { Pa } @ \mathbf{~} \mathbf{~ m})\end{array}$ \\
\hline 100 & 205 \\
\hline 200 & 202 \\
\hline 400 & 199 \\
\hline 800 & 196 \\
\hline
\end{tabular}

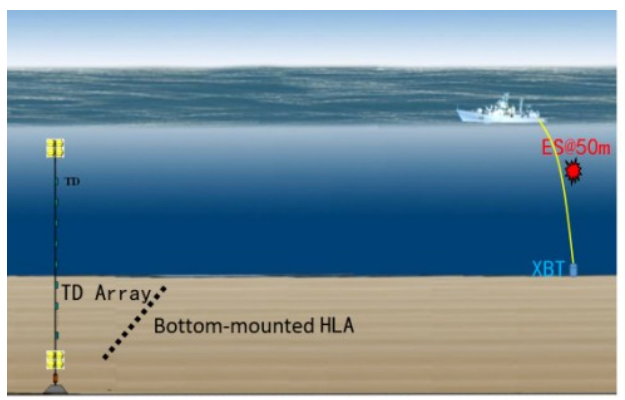

Fig. 2. Experimental configuration.

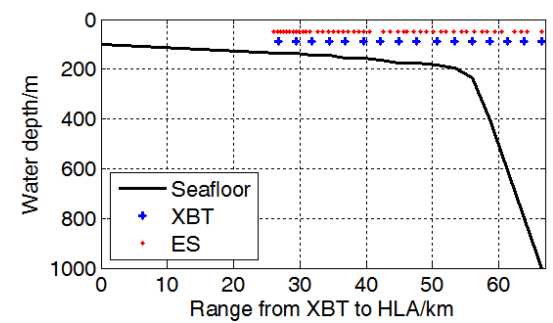

Fig. 3. Water depths and positions of ESs and XBTs casted along the propagation track. The HLA is at range of $0 \mathrm{~km}$.

\subsection{Data processing}

The sound speed profiles along the propagation track are shown in Fig.4, which are calculated based on the XBT data. The mean temperature profile measured by the vertical TD array near the HLA is shown in Fig.5. We see that there is obvious negative gradient in the speed/ temperature profiles all through the water column along the propagation track during the experiment.

The time-variation of the temperature profile near the HLA is shown in Fig.6, measured by the vertical TD array in 65 hours. It shows that there were linear internal waves around the HLA location during the experiment. But we could not find obvious non-linear internal waves in Fig.6.

Four received explosive signals are shown in Fig.7, of which the source-receiver distance are $57 \mathrm{~km}, 44 \mathrm{~km}$, $33 \mathrm{~km}$ and $26 \mathrm{~km}$ respectively.

The transmission loss (TL) of the received signals between $508 \mathrm{~Hz}$ and $640 \mathrm{~Hz}$ are calculated, as shown in Fig.8. The red dot-dash line in Fig.8, display name $\mathrm{TL}_{1}$, is the first order curve fitting result of TL. In this paper, the relative local energy intensity is calculated by 


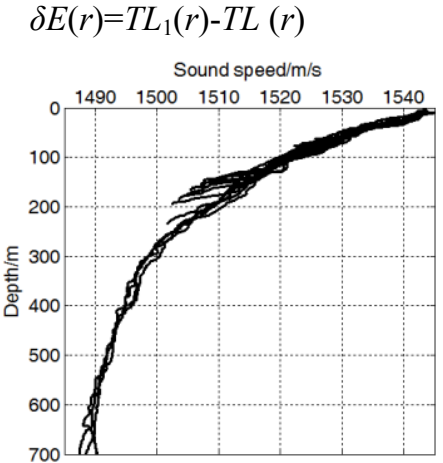

Fig. 4. The sound speed profiles measured by XBT.

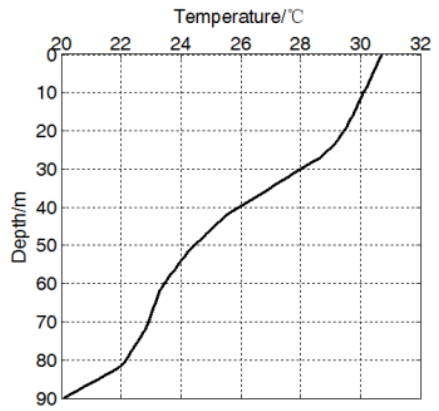

Fig. 5. The mean temperature profile near HLA.

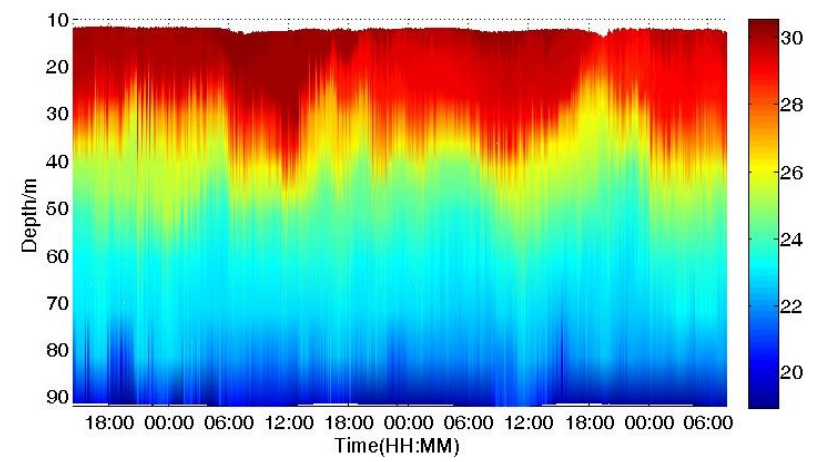

Fig. 6. Temperature profiles measured near the HLA location by the vertical TD array. Linear internal waves can be found.
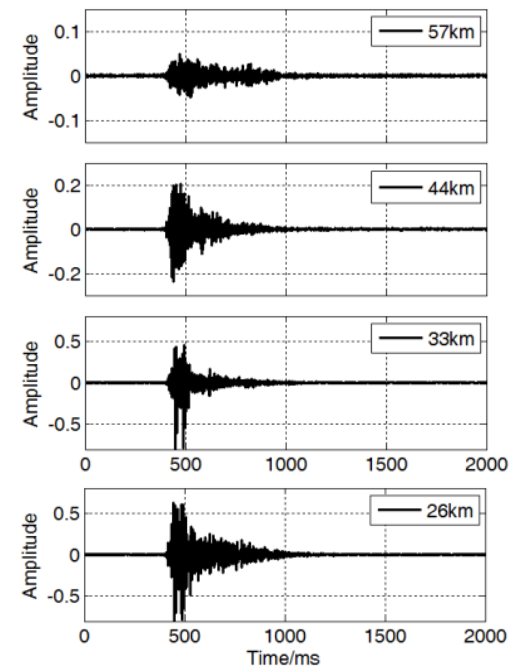

Fig. 7. Four received explosive signals (unfiltered).

The peaks on the curve of $\delta E$ versus source-receiver distance indicate local maximums of explosive soundfield intensity, as shown in Fig.9(b).
By Eq. (1), the experimental spatial correlation coefficients at different ranges are shown in Fig.9(a), while the explosive signals are processed in 508-640 Hz frequency band. As we can see in Fig.9, the transverse horizontal spatial correlation coefficient depends highly on the source-recerver range, and it fluctuates synchronously with the sound-field intensity while range varies. For example, the sound-field intensity has a local minimum at range of $31.4 \mathrm{~km}$ and a local maximum at range of $43.7 \mathrm{~km}$ (marked by red circle in Fig.9(b)). Correspondingly, the coefficient coefficient has a local minimum and maximum at $31.4 \mathrm{~km}$ and $43.7 \mathrm{~km}$ relatively. Fig.11 shows the curve of the correlation coefficient and its standard deviation with transverse separation varying at $31.4 \mathrm{~km}$ and $43.7 \mathrm{~km}$.

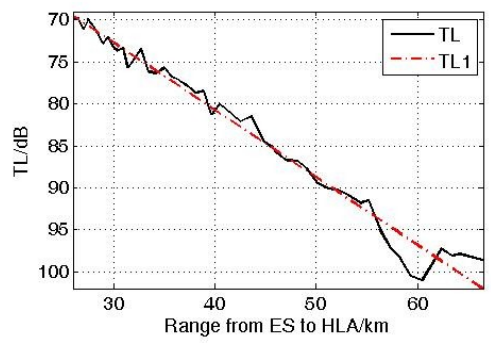

Fig. 8. Experimental $T L s$ at different ranges, $508 \mathrm{~Hz}-640 \mathrm{~Hz}$.

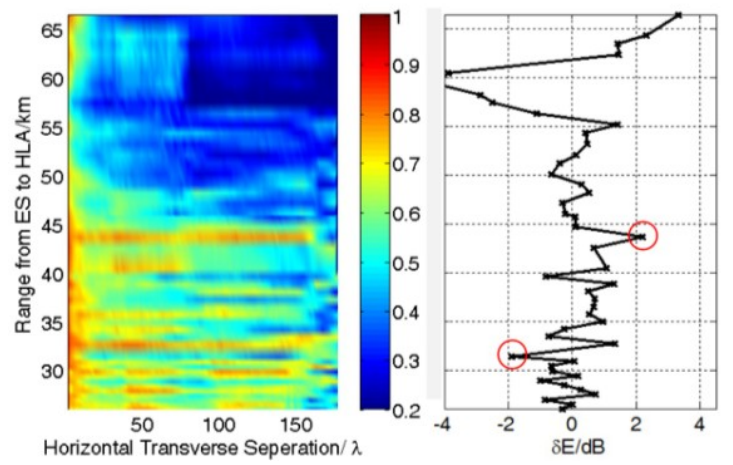

Fig. 9. Experimental spatial correlation coefficients (a) and $\delta E$ (b), $508 \mathrm{~Hz}-640 \mathrm{~Hz} . \delta E$ is relative local energy intensity.

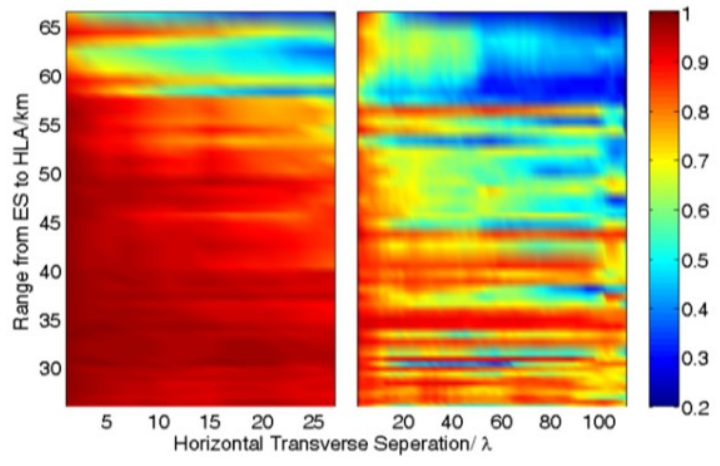

Fig. 10. Experimental spatial correlation coefficients. $80 \mathrm{~Hz}-$ $101 \mathrm{~Hz}(\mathrm{a}), 320 \mathrm{~Hz}-403 \mathrm{~Hz}$ (b).

The same phenomenon alse occures in other frequency bands, as indicated in Fig.10. This phenomenon is inconsistent with what Carey and Rouseff described in papers. Both of them argue that the transverse horizontal spatial coherence length depends only weakly on range. 
As for the 508 to $640 \mathrm{~Hz}$ frequency band, the transverse coherence length is shown to be larger than $170 \lambda$ (expressed in terms of certer-frequency wavelength) at most ranges less than $50 \mathrm{~km}$ in shallow water. It is much greater than Carey's shallow-water result $30 \lambda$ estimated from array signal gain after assuming a specific functional form for the coherence, but consistent with Rouseff's modeling result.
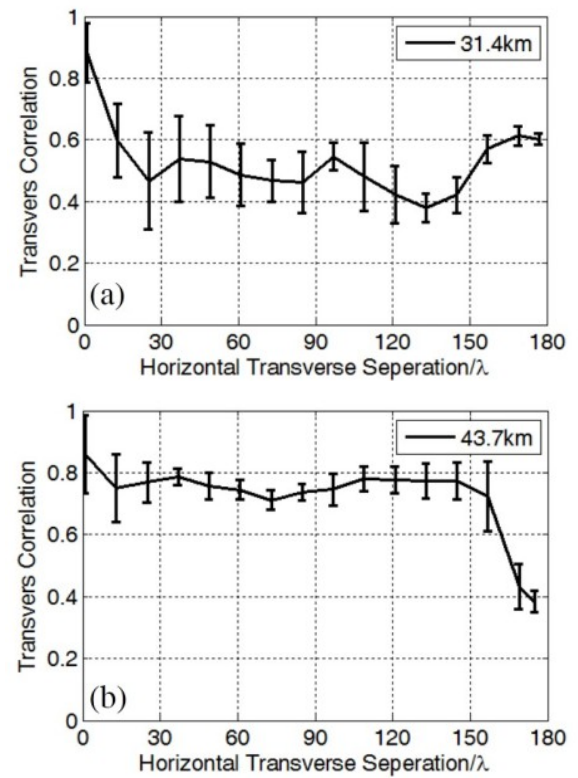

Fig. 11. Correlation coefficient and its error range at $31.4 \mathrm{~km}$ (a) and $43.7 \mathrm{~km}$ (b), $508 \mathrm{~Hz}-640 \mathrm{~Hz}$.

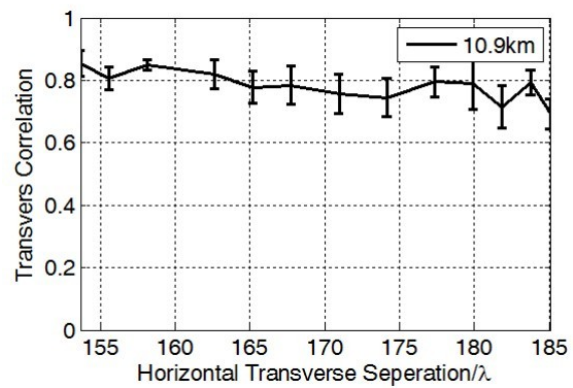

Fig. 12. Correlation coefficient and its error range at $10.9 \mathrm{~km}$ in $\operatorname{Exp} 2016,80 \mathrm{~Hz}-101 \mathrm{~Hz}$.

A similar experiment has been conducted in the same area of South China Sea in November 2016 (Exp2016). The bottom-mounted HLA with unequal spacing between adjacent elements had an aperture of about $3 \mathrm{~km}$. The spatial correlation coefficient and its standard deviation in $80-101 \mathrm{~Hz}$ frequency band are shown in Fig.12. Obviously, the transverse coherence length is larger than $185 \lambda$ at range of $10.9 \mathrm{~km}$. Only one explosive source was casted braodside to the large array, so the spatial correlation data at other ranges could not be obtained.

\section{Theoretical analysis}

In this section, a theoretical analysis will be made for the phenomenon of transverse horizontal correlation coefficient fluctuating with range.
The transverse horizontal correlation coefficient in the frequency domain can be written as

$$
\begin{aligned}
& \rho(r, \Delta r, \Delta l) \\
& =\max _{\tau} \frac{\left.\operatorname{Re}\left[\int_{\omega_{1}}^{\omega_{2}} P(r, l ; \omega) P^{*}(r+\Delta r, l+\Delta l ; \omega) e^{j \omega \tau} d \omega\right)\right]}{\sqrt{\int_{\omega_{1}}^{\omega_{2}}|P(r, l ; \omega)|^{2} d \omega \int_{\omega_{1}}^{\omega_{2}}|P(r+\Delta r, l+\Delta l ; \omega)|^{2} d \omega}}
\end{aligned}
$$

where $P(r, l ; \omega)$ is the spectrum of the sound pressure $p(r, l ; t), *$ represents the complex conjugation, $\omega$ denotes the angular frequency, $\omega_{1}$ and $\omega_{2}$ are the lower and upper angular frequencies.

For simplicity, let $\Delta r=0$ and $\omega_{1}=\omega_{2}$, so we have

$$
\rho(r, \Delta l)=\frac{\operatorname{Re}\left[P(r, l ; \omega) P^{*}(r, l+\Delta l ; \omega)\right]}{|P(r, l ; \omega)||P(r, l+\Delta l ; \omega)|}
$$

On condition that sound speed and density depend only on depth $z$, the underwater acoustic pressure field generated by a point source can be expressed by normal modes theory. The sound pressure can be written as

$$
P(r, l+\Delta l)=\sum_{m} A_{m} e^{j\left(\phi_{m}(r)+\theta_{m}(r, l+\Delta l)\right)}
$$

where $A_{m}$ and $\phi_{m}(r)+\theta_{m}(r, l+\Delta l)$ is the amplitude and the phase of the $\mathrm{m} t h$ mode respectively, $\phi_{m}(r)$ is deterministic phase and $\theta_{m}(r, l+\Delta l)$ is random phase caused by the uncertainty of ocean eviroment,

$$
\begin{gathered}
A_{m}=\frac{j}{\rho\left(z_{s}\right) \sqrt{8 \pi}} e^{-j \pi / 4} \Psi_{m}\left(z_{s}\right) \Psi_{m}\left(z_{r}\right) \frac{e^{j k_{m} r}}{\sqrt{k_{m} r}} \\
\phi_{m}(r)=k_{m} r \\
\theta_{m}(r, l+\Delta l)=\theta_{m}(r, l)+\delta \theta_{m}(r, \Delta l)
\end{gathered}
$$

where $\Psi_{m}\left(z_{s}\right)$ and $\Psi_{m}\left(z_{r}\right)$ are the eigenfunctions at the source and the receiver, respectively. $k_{m}$ is the horizontal wavenumber of the mth mode. $\delta \theta_{m}(r, \Delta l)$ is the mode phase transverse variation (MPTV).

According to Eq. (7)-(11), we have

$\rho(r, \Delta l)$

$=\sum_{m} \sum_{n} A_{m} A_{n} \cos \left(\phi_{m n}(r)-\delta \theta_{n}(r, \Delta l)\right) /|P(r, l)||P(r, l+\Delta l)|$

$=\left\{\sum_{m} \sum_{n} A_{m} A_{n}\left[\cos \left(\phi_{m n}(r)\right) \cos \left(\delta \theta_{n}(r, \Delta l)\right)+\right.\right.$

$\left.\left.\sin \left(\phi_{m n}(r)\right) \sin \left(\delta \theta_{n}(r, \Delta l)\right)\right]\right\} /\left[\sqrt{\sum_{m} \sum_{n} A_{m} A_{n} \cos \left(\phi_{m n}(r)\right)} \times\right.$

$\left.\sqrt{\sum_{m} \sum_{n} A_{m} A_{n}\left(\cos \left(\phi_{m n}(r)\right) \cos \left(\delta \theta_{m n}(r, \Delta l)\right)+\sin \left(\phi_{m n}(r)\right) \sin \left(\delta \theta_{m n}(r, \Delta l)\right)\right)}\right]$

where

$$
\begin{aligned}
& \phi_{m n}(r)=\phi_{m}(r)-\phi_{n}(r) \\
& \delta \theta_{m n}(r, \Delta l)=\delta \theta_{m}(r, \Delta l)-\delta \theta_{n}(r, \Delta l)
\end{aligned}
$$

By normal modes theory, the sound-field intensity fluctuates with range and depth caused by the 
interference between different modes. At some points in the sound field, the modes which make a major contribution to the total energy are almost in phase, so the sound-field intensity will have local peaks at these points in the rang-depth pattern. Similarly, the intensity will have local valleys at the points where the major modes are out of phase.

\subsection{Situation of modes in phase}

At the points where the major modes are in phase,

$$
\phi_{m}(r) \approx \phi_{n}(r)
$$

so we have

$$
\cos \left(\phi_{m n}(r)\right) \approx 1, \sin \left(\phi_{m n}(r)\right) \approx 0
$$

In the case of small transvers separation,

$$
\begin{aligned}
& \cos \left(\delta \theta_{n}(r, \Delta l)\right) \approx 1, \sin \left(\delta \theta_{n}(r, \Delta l)\right) \approx 0 \\
& \cos \left(\delta \theta_{m n}(r, \Delta l)\right) \approx 1, \sin \left(\delta \theta_{m n}(r, \Delta l)\right) \approx 0
\end{aligned}
$$

so we have

$$
\begin{aligned}
& \rho(r, \Delta l) \\
& \approx \sum_{m} \sum_{n} A_{m} A_{n} \cos \left(\phi_{m n}(r)\right) \cos \left(\delta \theta_{n}(r, \Delta l)\right) /\left(\sqrt{\sum_{m} \sum_{n} A_{m} A_{n} \cos \left(\phi_{m n}(r)\right)}\right. \\
& \left.\times \sqrt{\sum \sum A_{m} A_{n} \cos \left(\phi_{m n}(r)\right) \cos \left(\delta \theta_{m}(r, \Delta l)-\delta \theta_{n}(r, \Delta l)\right)}\right) \\
& \approx 1
\end{aligned}
$$

Besides, we can do the following analysis. The correlation coefficient of $P(r, l)$ and $P(r, \Delta l)$ can be written as

$$
\rho(r, \Delta l)=\cos (\Phi(r, l)-\Phi(r, l+\Delta l))
$$

where $\Phi(r, l)$ and $\Phi(r, l+\Delta l)$ is the phase angle of $P(r, l)$ and $P(r, l+\Delta l)$ respectively. In other words, the correlation coefficient is equal to the cosine of the angle between $P(r, l)$ and $P(r, l+\Delta l)$ on the complex plane. At the points where the major modes are in phase, the phases of the major modes are approximately equal, so small variation of mode phases, $\delta \theta_{m}(r, \Delta l)$, has little influence on the phase angle of the total sound field which is approximately the sum of the major modes, as shown in Fig.13. As a result,

$$
\Phi(r, l) \approx \Phi(r, l+\Delta l)
$$

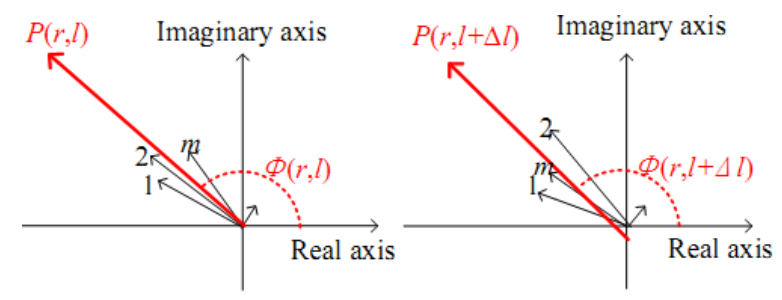

Fig. 13. Phase angle geometry. The major modes (mode 1, 2 and $\mathrm{m}$ ) have similar phases. Small variation of mode phases has little influence on the phase angle of their sum.
So $\rho(r, \Delta l)$ is approximately equal to 1 at these points.

\subsection{Situation of modes out of phase}

At the points where the major modes are out of phase, the phases of the major modes are dispersed from 0 to $2 \pi$, so the cosine and sine of $\phi_{m m}(r)$ are random variables in the range of -1 to 1 , and Eq. (16) is no longer valid. In this case, it is difficult to simplify Eq. (12) as we do in Section 4.1.

However, we can still analyse the correlation coefficient from the perspective of geometry. As shown in Fig.14, the phases of the major modes are dispersed from 0 to $2 \pi$, so small variation of mode phases has great influence on the phase angle of their sum. In a statistical sense, the value of $\Phi(r, l)-\Phi(r, l+\Delta l)$ is distributed randomly in range of 0 to $2 \pi$. So we can deduce directly

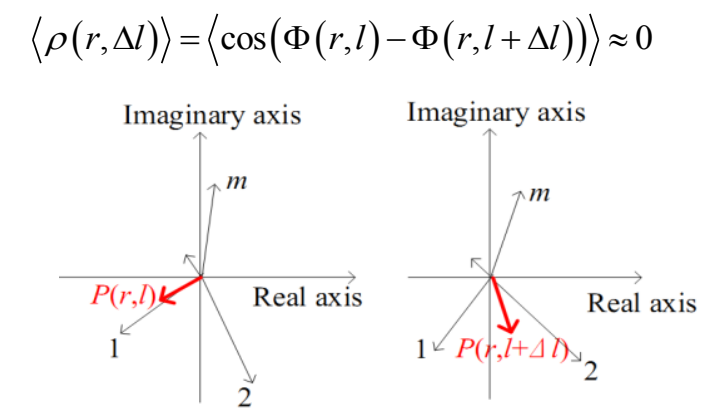

Fig. 14. Phase angle geometry. Phases of major modes (mode 1,2 and $\mathrm{m}$ ) are dispersed. Small variation of mode phases has great influence on the phase angle of their sum.

\subsection{Simulation}

We use the normal mode program KrakenC to calculate the acoustic field at $100 \mathrm{~Hz}$. In the simulation, the depth of water column is $100 \mathrm{~m}$, the sound speed profile is calculated according to the temperature in Fig.5 and thought to be range-independent. The source/receiver depth is $50 \mathrm{~m} / 100 \mathrm{~m}$. A two-layer liquid bottom model is used and the thickness of the sediment layer is $10 \mathrm{~m}$. The sound speed is $1550 \mathrm{~m} / \mathrm{s}$, the density is $1.6 \mathrm{~g} / \mathrm{cm}^{3}$, and the attenuation coefficient is $0.09 \mathrm{~dB} / \lambda$ inside the sediment layer. The infinite basement has a sound speed of $1650 \mathrm{~m} / \mathrm{s}$, a density of $2.0 \mathrm{~g} / \mathrm{cm}^{3}$, and an attenuation coefficient of $0.18 \mathrm{~dB} / \lambda$.

Fig.15(a) shows the simulated $T L s$ at ranges from $50 \mathrm{~km}$ to $53 \mathrm{~km}$.

For simplicity, the transverse variation, $\delta \theta_{m}(r, \Delta l)$, of each mode phase caused by the variation of ocean environment is assumed to be subjected to uniform distribution and to be independent and identical distribution. On these assumptions, the average correlation coefficient of 200 Monte-Carlo simulation results is shown in Fig.15(b), of which the $y$ axis is the standard deviation of MPTV, $\delta \theta_{m}(r, \Delta l)$. Compared with Fig.15(a), the correlation coefficient is shown to depend highly on range, and it fluctuates synchronously with the sound-field intensity while range varies. 
Fig.15(c) shows the simulated weighted average phase difference (WAPD) at ranges from $50 \mathrm{~km}$ to $53 \mathrm{~km}$. Here, WAPD is defined as

$$
W A P D(r)=\sum_{m} \sum_{n}\left|A_{m} A_{n}\right|^{2} \phi_{m n}(r) / \sum_{m} \sum_{n}\left|A_{m} A_{n}\right|^{2}
$$

where $\phi_{m}(r)$ is deterministic phase as Eq. (10).

In Fig.15, WAPD has local minimum at range of $50 \mathrm{~km}$ and $52 \mathrm{~km}$, where the sound-field intensity and the standard deviation of MPTV have local maximums, as analysed in Section 4.1; WAPD has local maximums at range of $50.9 \mathrm{~km}$ and $52.7 \mathrm{~km}$, where the sound-field intensity and the standard deviation of MPTV have local minimums, as analysed in Section 4.2.

It needs to be pointed out that the fluctuation of correlation coefficient with range is not due to the fluctuation of MPTV, $\delta \theta_{m}(r, \Delta l)$. Generally speaking, $\delta \theta_{m}(r, \Delta l)$ changes slowly with range $r$. Even if the standard deviation of MPTV keeps constant with range $r$, the average correlation coefficient still fluctuates with $r$, as shown in Fig.15(b). The experimental result and theoretical analysis indicate that the interference between normal modes is the fundamental cause of the fluctuation of correlation coefficient and acoustic intensity with range. Rouseff's modelling result of the transverse horizontal coherence length depends weakly on range, because of "neglecting the $m \neq m$ ' cross terms" (See Eq. (19) in Ref. [1]).

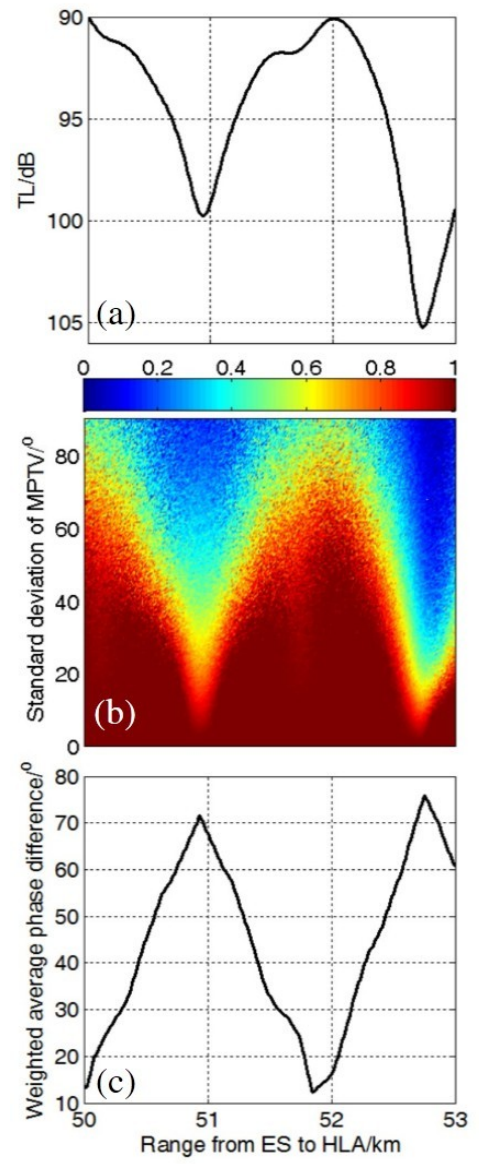

Fig. 15. Simulation results at $100 \mathrm{~Hz}$. (a) $\boldsymbol{T} \boldsymbol{L}$ versus range. (b) Average correlation coefficient of 200 Monte-Carlo simulations. (c) Weighted average phase difference versus range.

\section{Conclusions and perspectives}

The transverse horizontal spatial coherence in shallow water is investigated by using the experimental data obtained in the Northern South China Sea and by combining the normal modes theory with geometry analysis. It is shown that the transverse horizontal spatial coherence depends highly on the source-receiver range, which fluctuates synchronously with the sound-field intensity while range varies, and that the fundamental cause of this phenomenon is the interference between normal modes. At the ranges where the major modes are in phase, the transverse coherence is strong locally as well as the acoustic intensity, and the converse is also true.

What's more, the experimental results showed that the transverse coherence length was larger than $170 \lambda$ at most ranges less than $50 \mathrm{~km}$ in shallow water as for the 508 to $640 \mathrm{~Hz}$ frequency band, and was larger than $185 \lambda$ at a range of $10.9 \mathrm{~km}$ in shallow water as for the 80 to $101 \mathrm{~Hz}$ frequency band. These results of coherence length indicate that it is possible to utilize large aperture (e.g. 200 $\lambda$ ) horizontal line array for coherent processing in shallow water. This is of great significance for long distance acoustic detection and communication in shallow water.

The obvious degradation of the correlation coefficient when the source is dropped in the area with strong slope sea-floor, as shown in Fig.3, Fig.9(a) and Fig.10, and the major environmental reasons leading to coherence degradation with transverse separation in shallow water will be investigated in the future.

The authors thank Dr. Guihua Ji for her help in providing the TD array data during the 2017 experiment. The authors thank all the researchers for their help in the experiments in 2016 and 2017. The work was supported by the Foundation of Chinese Academy of Sciences (No.6141A01051601) and IACAS Young Elite Researcher Project (No.QNYC201742).

\section{References}

1. D. Rouseff and A. A. Lunkov, J. Acoust. Soc. Am. 138, 2256 (2015)

2. L. H. Guo, Gong Z X and L X Wu, Chin. Phys. Lett. 181366 (2001)

3. X. X. Su, Li F H and S S Jian, Tech. Acoust. 26579 (2007, in Chinese)

4. L. Wan et al, Acoust. Phys. 55383 (2009)

5. Q. Wang and R. H. Zhang, J. Acoust. Soc. Am. 92 932 (1992)

6. F. H. Li and R. H. Zhang, Chin. Phys. Lett. 252539 (2008)

7. J. Li, Z. L. Li and Y. Ren, Chin. Phys. B 25124310 (2016)

8. W. M. Carey, J. Acoust. Soc. Am. 104831 (1998)

9. Y. J. Zhang, R. H. Zhang and F. H. Li, Chin. Phys. Lett. 27084201 (2010) 